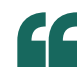

What is really cool about the technique is that we can see how different the sites really are
METAL CLUSTERS

\section{MAD from the scatter}

Biological electron transport and catalysis is often mediated by metal clusters, on which the impacts of ligand coordination and redox can be softened by distribution over multiple metal centres. There are ways to measure relative redox levels within a cluster, but it is harder to assign each level to a particular metal site. A team led by Ted Betley has now addressed this problem by studying a series of well-defined triiron complexes with multi-wavelength anomalous diffraction (MAD). Writing in the Proceedings of the National Academy of Sciences, the team describe how symmetry, electronic delocalization and metal-metal bonding affect MAD data. Their work will enable us to better interpret MAD data on more complicated systems such as metalloenzymes.

The redox state of a metal in a mononuclear complex can be probed by collecting X-ray absorption near-edge spectroscopy (XANES)

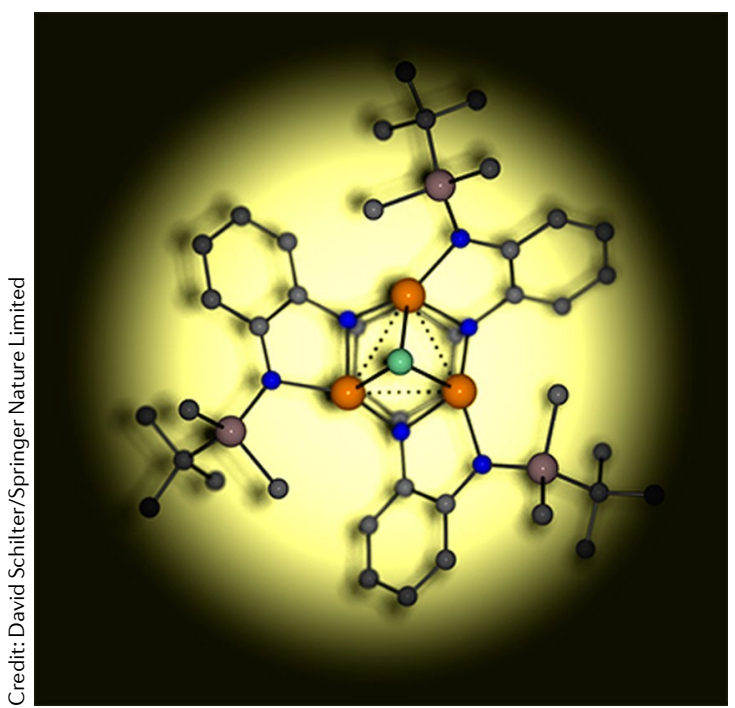

or Mössbauer spectroscopy data and comparing them with data from complexes with unambiguous redox states and bonding. Multinuclear complexes, however, can afford spectra with multiple signals that may each be difficult to assign to a specific site; but, if a complex is available as single crystals, it can be subjected to a series of diffraction experiments in which data are collected at X-ray energies near the absorption edges of the metals of interest. "Although conventional $\mathrm{X}$-ray diffraction is done far from the absorption edges of the elements investigated, absorption and scattering components of atomic scattering factors vary substantially near the absorption edge," explains Betley. "Thus, refining scattering factors as a function of incident X-ray energy allows you to get remarkable site-specific redox information within clusters."

Taking $\left[\mathrm{Fe}_{3} \mathrm{~L}\right.$ (pyridine)] as a model compound, in which $\mathrm{L}^{6-}$ is a hexaamido ligand derived from cis,cis-1,3,5-triaminocyclohexane, Betley and colleagues also prepared $\left[\mathrm{Fe}_{3} \mathrm{~L}\left(\mu^{3}-\mathrm{Cl}\right)\right]^{-}$(shown in the image), $\left[\mathrm{Fe}_{3} \mathrm{~L}\left(\mu^{3}-\mathrm{NPh}\right)\right]^{2-}$ and $\left[\mathrm{Fe}_{3} \mathrm{~L}\left(\mu^{3}-\mathrm{NPh}\right)\right]$ to get a series of complexes that differ in terms of symmetry, redox, $\mathrm{Fe}-\mathrm{Fe}$ bonding and ligation. After collecting full diffraction data from each complex using $30.5 \mathrm{keV} \mathrm{X}$-rays, the team performed MAD by collecting several partial datasets in the range of $7.085 \mathrm{keV}$ to $7.150 \mathrm{keV}$ - the region around the Fe K-edge (the energy required to eject a 1s electron). Using the atomic coordinates obtained from the full dataset, they refined each partial dataset only in terms of Fe scattering factors and arrived at a plot of real scattering factor $\left(f^{\prime}\right)$ versus energy for each Fe in each complex. The minimum of such an $f^{\prime}$ plot corresponds to a XANES edge, with the benefit that one knows which $\mathrm{Fe}$ gives rise to the spectral feature. For the almost $C_{3}$-symmetric complex $\left[\mathrm{Fe}_{3} \mathrm{~L}\left(\mu^{3}-\mathrm{Cl}\right)\right]^{-}$, the three $f^{\prime}$ plots overlap, indicating that each $\mathrm{Fe}^{\mathrm{II}}$ site is equivalent. By contrast, the asymmetric complex $\left[\mathrm{Fe}_{3} \mathrm{~L}\right.$ (pyridine)] also has three formally $\mathrm{Fe}^{\mathrm{II}}$ sites but gives rise to $f^{\prime}$ minima at energies spanning a $10 \mathrm{eV}$ range - almost as wide as the range previously observed for $\mathrm{Fe}^{0}$ all the way to $\mathrm{Fe}^{\mathrm{III}}$. "What is really cool about the technique is that we can see how different the sites really are even though they are formally at the same oxidation level," notes Betley. "The analysis revealed how simple perturbations can have a substantial impact on relative oxidation levels within clusters."

Although MAD is sensitive to metal-ligand coordination geometry, it can distinguish the $\mathrm{Fe}^{\mathrm{II}}{ }_{3}$ complex $\left[\mathrm{Fe}_{3} \mathrm{~L}\left(\mu^{3}-\mathrm{NPh}\right)\right]^{2-}$ from the formally $\mathrm{Fe}_{2}{ }_{2} \mathrm{Fe}^{\text {II }}$ species $\left[\mathrm{Fe}_{3} \mathrm{~L}\left(\mu^{3}-\mathrm{NPh}\right)\right]$, with the latter giving rise to overlapping $f^{\prime}$ plots diagnostic of a symmetric species featuring $\mathrm{Fe}-\mathrm{Fe}$ bonding. This systematic study of model compounds demonstrates the utility of MAD but also shows its limitations in terms of covalenceinduced $f^{\prime}$ broadening. Thus, it is only with a set of structurally relevant model compounds and a deep understanding that we can exploit MAD to unravel the structures of elaborate multinuclear systems.

David Schilter

ORIGINAL ARTICLE Bartholomew, A. K. et al. Exposing the inadequacy of redox formalisms by resolving redox inequivalence within isovalent clusters. Proc. Natl Acad. Sci. USA https://doi.org/ 10.1073/pnas.1907699116 (2019) 